\title{
A necessary condition for the well posed hyperbolic mixed problem with variable coefficients
}

\author{
By \\ Kunihiko KaJITANI \\ (Communicated by Prof. Mizohata June 29, 1973)
}

\section{$\S 1$. Introduction}

In the present article we shall derive a necessary condition (say, Lopatinski's condition) for the well posed mixed problem of the first order hyperbolic systems with variable coefficients. Here we say that the mixed problem is well posed, if this problem has a local solution for any sufficiently smooth deta and a finite propagation speed.

In the case of constant coefficients, R. Hersh in [5] and T. Kasahara in [10] proved the global existence of the solution for the mixed problem of the first order hyperbolic systems under Lopatinski's condition, and Hersh in [6] and [7] researched into the existence and the finite propagation speed of the solution for the higher order hyperbolic systems. Moreover T. Shirota in [14] studied precisely the finite propagation speed of the solution for the single higher order hyperbolic equations. Recently R. Sakamoto in [13] characterized completely the necessary and sufficient conditon for the existence and the finite propagation speed of the solution for the single higher order hyperbolic equations.

In the case of variable coefficients, M. Ikawa proved in [3] that the mixed problem for the wave equation with an oblique derivative boundary condition is well posed. J. Chazarain in [2] and R. Beals in [1] proved the existence of the solution in Gevrey-class for the 
mixed problem for hyperbolic systems (not necessary strictly hyperbolic) by use of semigroup theory and derived a finite propagation speed. In [7] the auther showed a necessary condition for the well posed mixed problem for $2 \times 2$ hyperbolic systems with analytic coefficients. In this article we shall extend the result obtained in [7] to the general systems. Namely, we shall prove that Lopatinski's contidion for the principal part of the hyperbolic systems considered and for the boundary condition is necessary under some assumptions, if the mixed problem for the systems is well posed. It is seemed that this problem is corresponding to those which $\mathrm{P}$. Lax in [11] and S. Mizohata in [12] studied for the Cauchy problem.

We consider the following mixed problem in $\Omega$ a neighbourhood of the origin in $R_{+}^{k} \times(0, \infty)$

$$
\begin{gathered}
L[u]=\frac{\partial}{\partial t}-\sum_{j=1}^{k} A_{j}(x, t) \frac{\partial}{\partial x_{j}} u-B(x, t) u=f(x, t),(x, t) \text { in } \Omega \\
u(x, 0)=g(x), \quad x \text { in } D=\Omega \cap\{t=0\}, \\
P\left(x^{\prime}, t\right) u\left(x^{\prime}, 0, t\right)=h\left(x^{\prime}, t\right), \quad\left(x^{\prime}, t\right)=\left(x_{1}, \ldots, x_{k-1}, t\right) \\
\text { in } G=\Omega \cap\left\{x_{k}=0\right\},
\end{gathered}
$$

where $A_{j}(x, t)$ and $B(x, t)$ are $m \times m$ matrices of infinitely differentiable elements, $u(x, t), f(x, t)$ defined in $\Omega$ and $g(x)$ defined in $D$ are vector valued functions of $k$ components, $P\left(x^{\prime}, t\right)$ is a $l \times m$ matrix of infinitely differentiable elements and $h(x, t)$ is a vector valued function defined in $G$ of $l$ components.

We pose the following assumptions,

[A. I] $L$ is hyperbolic at origin, that is the characteristic matrix $\sum_{j=1}^{k} A_{j}(0,0) \eta_{j}$ has only real eigen values for any $\left(\eta_{1}, \ldots, \eta_{k}\right)$ in $R^{k}-\{0\}$. $A_{k}(0,0)$ is non singular.

[A. II] the rank of $P(0,0)$ is equal to $l$ and $l$ is the number of negative eigen values of $A_{k}(0,0)$.

We put $M(\lambda, i \eta)=A_{k}^{-1}(0,0)\left(\lambda-\sum_{j=1}^{k-1} A_{j}(0,0) \eta_{j}\right)$ then it follows from hyperbolicity of $L$ that the real part of eigen values of $M(\lambda, i \eta)$ is 
not zero for $\operatorname{Re} \lambda>0, \eta$ in $R^{k-1}$ and that the number of these eigen values with negative real part is equal to $l$. We define by $E^{-}(\lambda, \eta)$ (resp. $E^{+}(\lambda, \eta)$ ) the generalized eigen space corresponding to eigen values with negative (resp. positive) real part of $M(\lambda, \eta)$. We define

$$
Q^{ \pm}(\lambda, \eta)=\frac{1}{2 \pi i} \oint_{\Gamma_{ \pm}}(\zeta-M)^{-1} \mathrm{~d} \zeta
$$

where $\Gamma_{+}\left(\right.$resp. $\left.\Gamma_{-}\right)$is a Jordan's curve containing only eigen values with positive (resp. negative) real part. We define $\Lambda(\lambda, \eta)$,

$$
\Lambda(\lambda, \eta)=\frac{1}{2 \pi i} \oint_{r_{+}}(\zeta-M)^{-1} A_{k}^{-1}(0,0)(\zeta-M)^{-1} Q^{-} d \zeta
$$

[A. III] (i) $E^{-}(\lambda, \eta)$ consists of only eigen vectors of $M(\lambda, \eta)$ (has not generalized eigen vectors) for $\operatorname{Re} \lambda>0, \eta$ in $R^{k-1}$.

(ii) $\left\{E^{-}(\lambda, \eta) \cup\left(\operatorname{Ker} P(0,0) \cap E^{+}(\lambda, \eta)\right)\right\} \cap\left\{\Lambda(\lambda, \eta)\left(E^{-}(\lambda, \eta) \cap \operatorname{Ker} P(0,0)\right)\right\}=$ $\{0\}$. and $\operatorname{rank} \Lambda(\lambda, \eta) \geq \operatorname{dim}\left(E^{-}(\lambda, \eta) \cap \operatorname{Ker} P(0,0)\right)$, for $\operatorname{Re} \lambda>0, \eta$ in $R^{k-1}$.

Remark 1. We put $E_{0}^{ \pm}(\lambda, \eta)=\operatorname{Ker} F(0,0) \cap E^{ \pm}(\lambda, \eta)$ and $E_{1}^{ \pm}(\lambda, \eta)$ $=\left\{h \in E^{ \pm}(\lambda, \eta)\right.$ and $\left.h \notin E_{0}^{ \pm}(\lambda, \eta)\right\}$. Then (ii) of [A. III] is equivalent (ii) $\Lambda$ is an one to one and onto map from $E_{0}^{-}(\lambda, \eta)$ to $E_{1}^{+}(\lambda, \eta)$ for $\operatorname{Re} \lambda>0, \eta$ in $R^{k-1}$.

Remark 2. In the case of $m=2$ (cf. [7]), (ii) or (ii) ${ }^{\prime}$ is valid, if and only if it holds that for $\operatorname{Re} \lambda>0$,

$$
E^{-}(\lambda, 0) \cap \operatorname{Ker} P(0,0)=\{0\}
$$

We say that (1.1) has a finite propagation speed, if there exist a positive number $\delta_{0}$ and $\left(x_{0}, t_{0}\right)$ such that (1.1) has the unique solution in $\Omega\left(x_{0}, t_{0}\right)=\left\{(x, t) ;\left|x-x_{0}\right|<\delta_{0}\left(t_{0}-t\right), x_{k}>0, t>0\right\}$ for any $\left(x_{0}, t_{0}\right)$, $x_{0 k}>0, t>0$, in a neighbourhood of the origin.

Definition 1. We say that the mixed problem (1.1) is well posed in a neighbourhood of the origin, if for any deta $\left\{f(x, t), g(x), h\left(x^{\prime}\right.\right.$, $t)\}$ infinitely differentiable in a neighbourhood of the origin, with the 
compatibility condition, there exists a neighbourhood $\Omega$ of the origin such that (1.1) has a solution $u(x, t)$ in $C^{\infty}(\bar{\Omega})$ and has a finite propagation speed.

Definition 2. We denote by $L_{0}$ the principal part of $L$, that is, $L_{0}=\frac{\partial}{\partial t}-\sum_{j=1}^{k} A_{j}(x, t) \frac{\partial}{\partial x_{j}}$. We say that $\left(L_{0}, P\right)$ satisfies Lopatinski's condition at the origin, if it holds $E^{-}(\lambda, \eta) \cap \operatorname{Ker} P(0,0)=\{0\}, \quad$ for $\operatorname{Re} \lambda>0, \quad \eta$ in $R^{k-1}$.

Now we state our result,

Theorem. Suppose that [A. I], [A. II] and [A. III] are valid. Then $\left(L_{0}, P\right)$ satisfies Lopatinski's condition at the origin, if the mixed problem (1.1) is well posed in a neighbourhood of the origin.

Remark 3. If we do not assume (ii) of [A.III], our theorem does not generally hold. For example $(m=2, k=2$ and $l=1)$, we put

$$
\begin{aligned}
& L=\frac{\partial}{\partial t}-\left(\begin{array}{ll}
1 & 0 \\
0 & 1
\end{array}\right) \frac{\partial}{\partial x_{1}}-\left(\begin{array}{rr}
-1 & 0 \\
0 & 1
\end{array}\right) \frac{\partial}{\partial x_{2}}-\left(\begin{array}{ll}
0 & 1 \\
1 & 0
\end{array}\right), \\
& P=(0,1) .
\end{aligned}
$$

We can see easily that this example does not satisfy (1.3) and (1.4). But the mixed problem for $(L, B)$ is well posed (cf. [13]). In the case of constant ocefficients, (1.1) is well posed and (1.3) is valid, if and only if $\left(L_{0}, P\right)$ satisfies (1.4) (cf. [6], [13] and [14]).

To prove our theorem we shall apply to the mixed problem the method of the asymptotic solution which P. D. Lax in [11] used for the Cauchy problem.

\section{§2. Proof of Theorem}

We shall prove our theorem by contradiction. To do so, we need an inequality derived by the closed graph theorem. We denote $\Omega$ $\left(x_{0}, t_{0}\right)=\left\{(x, t) ;\left|x-x_{0}\right| \leq \delta\left(t_{0}-t\right) ; 0 \leq t \leq t_{0}, x \in R_{+}^{k}\right\}$, where $x_{0}$ in $R_{+}^{k}$ and $t_{0}>0$ are fixed, and $\delta$ is a positive constant. Then, if the mixed problem is well posed in a neighbourhood of the origin, it follows 
from the closed graph theorem that for any $\left(x_{0}, t_{0}\right)$ in a neighbourhood of the origin and for any $u(x, t)$ in $C^{\infty}\left(\Omega\left(x_{0}, t_{0}\right)\right)$ there exist a positive constant $C\left(x_{0}, t_{0}\right)$ and a positive integer $s_{0}$ such that

$$
\begin{gathered}
|u|_{0, \Omega\left(x_{0}, t_{0}\right)} \leqslant C\left(x_{0}, t_{0}\right)\left\{|L u|_{s_{0}, \Omega\left(x_{0}, t_{0}\right)}+|u|_{s_{0}, D\left(x_{0}, t_{0}\right)}\right. \\
\left.+|P u|_{s_{0}, G\left(x_{0}, t_{0}\right)}\right\}
\end{gathered}
$$

where $D\left(x_{0}, t_{0}\right)=\Omega\left(x_{0}, t_{0}\right) \cap\{t=0\}$ and $G\left(x_{0}, t_{0}\right)=\Omega\left(x_{0}, t_{0}\right) \cap\left\{x_{k}=0\right\}$. We note that $C\left(x_{0}, t_{0}\right)$ may be generally dependent of $\left(x_{0}, t_{0}\right)$. But we can see a behaviour of $C\left(x_{0} n^{-1}, t_{0} n^{-1}\right)$ for $n \rightarrow \infty$. We wtire $\Omega(n)=$ $\Omega\left(x_{0} n^{-1}, t_{0} n^{-1}\right), D(n)=D\left(x_{0} n^{-1}, t_{0} n^{-1}\right)$ and $G(n)=G\left(x_{0} n^{-1}, t_{0} n^{-1}\right)$. Then we have,

Lemma 2.1. Suppose that the mixed problem (1.1) is well posed in a neighbourhood of the origin. Then for any positive integer $n$ there exist a positive constant $C_{0}$ and positive integers $s_{0}$ and $s_{1}$ (independent of $n)$ such that for any $u(x, t)$ in $C^{\infty}\left(\Omega\left(x_{0}, t_{0}\right)\right.$ ),

$$
|u|_{0, \Omega(n)} \leqslant C_{0} n^{s_{1}}\left\{|L u|_{s_{0}, \Omega(n)}+|u|_{s_{0}, D(n)}+|P u|_{s_{0}, G(n)}\right\}
$$

We can derive this lemma from (2.1) by the same method as Lemma 2.1 in [9].

We put

$$
M(n)=A_{k}^{-1}\left(n^{-1} x, n^{-1} t\right)\left\{n \frac{\partial}{\partial t}-n \sum_{j=1}^{k} A_{j}\left(n^{-1} x, n^{-1} t\right) \frac{\partial}{\partial x_{j}}-B\left(n^{-1} x, n^{-1} t\right)\right\}
$$

and

$$
P(n)=P\left(n^{-1} x^{\prime}, n^{-1} t\right)
$$

Then we have,

Lemma 2.2. Suppose that the mixed problem (1.1) is well posed in a neighbourhood of the origin. Then there exist a positive constant $C_{1}$ and positive integers $s_{0}$ and $s_{1}$ such that for any positive integer $n$ and for any $u(x, t)$ in $C^{\infty}\left(\Omega\left(x_{0}, t_{0}\right)\right)$, 


$$
\begin{aligned}
&|u|_{0, \Omega(x, t)} \leqslant C_{1} n^{s_{1}+s_{0}}\left\{|M(n) u|_{s_{0}, \Omega\left(x_{0}, t\right)}+|u|_{s_{0}, D\left(x_{0}, t_{0}\right)}\right. \\
&\left.+|P(n) u|_{s_{0}, G\left(x_{0}, t_{0}\right)}\right\}
\end{aligned}
$$

Proof. This lemma was suggested by V.J. Ivrii [4]. We apply the inequality $(2.2)$ to $u_{n}(x, t)=u(n x, n t)$. We obtain easily (2.3) under the change of variables, $y=n x$ and $s=n t$. q.e.d.

Proof of Theorem. Suppose that $\left(L_{0}, P\right)$ does not satsify the Lopatinski's condition, that is, there exist linearly independent vectors $z_{1}, \ldots, z_{l^{\prime}}$ in $C^{m}$ and $\lambda_{0}, \operatorname{Re} \lambda_{0}>0, \eta_{0}$ in $R^{k-1}$ such that

$$
\left\{z_{1}, \ldots, z_{l^{\prime}}\right\} \subset E^{-}\left(\lambda_{0}, \eta_{0}\right) \cap \operatorname{Ker} P(0,0)
$$

We denote

$$
M^{0}=A_{k}^{-1}(0,0)\left(\lambda_{0}-i \Sigma A_{j}(0,0) \eta_{j}\right)
$$

Let $\xi_{j}^{-}, j=1, \ldots, l$ be the eigen values with a negative real part of $M^{0}$ and $h_{j}$ (resp. $\omega_{j}$ ) the right (resp. left) eigen vectors corresponding to $\xi_{j}^{-}$. For simplicity, we assume that $\xi_{j}^{-}, j=1, \ldots, l$, are simple eigen values.

We shall construct the asymptotic solution of (1.1) having the following form,

$$
u(x, t)=\sum_{j>0} n^{-j} \sum_{p=1}^{l} \exp \left\{n\left(\xi_{p}^{-} x_{k}+\lambda_{0} t-i x^{\prime} \eta_{0}\right)\right\} u_{j}^{(p)}(x, t),
$$

here, $u_{j}^{(p)}$ are functions to be determined.

Applying $M(n)$ to (2.5), we have,

$$
\begin{gathered}
M(n)[u]=\sum_{p=1}^{l}\left[n^{2}\left(\xi_{p}^{-}-M^{0}\right) u_{0}^{(p)}+n\left\{\left(\xi_{p}^{-}-M^{0}\right) u_{1}^{(p)}\right.\right. \\
\left.-\left(\frac{\partial}{\partial x_{k}}-M_{0}\left(D_{t}, D_{x}\right)-M_{1}\left(x, t ; \lambda_{0}, \eta_{0}\right)\right) u_{0}^{(p)}\right\}+\cdots \\
+n^{-j+2}\left\{\left(\xi_{p}^{-}-M^{0}\right) u_{j}^{(p)}+\left(\frac{\partial}{\partial x_{k}}-M_{0}\left(D_{t}, D_{x}\right)\right.\right. \\
\left.-M_{1}\left(x, t ; \lambda_{0}, \eta_{0}\right)\right) u_{j-1}^{(p)}
\end{gathered}
$$




$$
\begin{aligned}
& \left.-\sum_{\substack{i+s=j \\
s<j=1}} M_{i}\left(x, t ; \lambda_{0}, i \eta_{0}\right) u_{s}^{(p)}-\sum_{\substack{i+s=j-1 \\
s<j-1}} M_{i}\left(x, t, D_{t}, D_{x^{\prime}}\right) u_{s}^{(p)}\right\} \\
& +\cdots] \exp \left\{n\left(\xi_{p}^{-} x_{k}+\lambda_{0} t-i x^{\prime} \cdot \eta_{0}\right)\right\}
\end{aligned}
$$

where,

$$
\begin{aligned}
& M_{0}\left(D_{t}, D_{x^{\prime}}\right)=A_{k}^{-1}(0,0)\left(\frac{\partial}{\partial t}-\sum_{j=1}^{k-1} A_{j}(0,0) \frac{\partial}{\partial x_{j}}\right), \\
& M_{j}\left(x, t, D_{t}, D_{x^{\prime}}\right)=\sum_{|v|+i=j} x^{v} t^{i} M_{i v}\left(\frac{\partial}{\partial t}, \frac{\partial}{\partial x^{\prime}}\right)+\sum_{|v|+i=j-1} x^{v} t^{i} B_{v i},
\end{aligned}
$$

and operating $P(n)$ to $u$, we get

$$
\begin{aligned}
P(n) & {\left.[u]\right|_{x_{k}=0}=\sum_{p=1}^{l} \exp n\left(\lambda_{0} t-i x^{\prime} \eta_{0}\right)\left[\left.P_{0} u_{0}^{(p)}\right|_{x_{k}=0}\right.} \\
& +\left.n^{-1}\left\{P_{0} u_{1}^{(p)}+P_{1}(x, t) u_{0}^{(p)}\right\}\right|_{x_{k}=0}+\cdots \\
& \left.+\left.n^{-j}\left\{P_{0} u_{j}^{(p)}+\sum_{i+s=j-1} P_{i}\left(x^{\prime}, t\right) u_{s}^{(p)}\right\}\right|_{x_{k}=0}+\cdots\right]
\end{aligned}
$$

where $P_{0}=P(0,0)$,

$$
P_{j}\left(x^{\prime}, t\right)=\sum_{|v|+i=j} x^{\prime}{ }^{v} t^{i}(v ! i !)^{-1}\left(\left(\frac{\partial}{\partial x^{\prime}}\right)^{v}\left(\frac{\partial}{\partial t}\right)^{i} P\right)(0,0) .
$$

Thus if we work out formally the coefficients of $n^{-j}$ in (2.6) and (2.7) and set each of them equal to zero, we obtain the sequence of the recursion formulas,

$$
\begin{aligned}
& \left(\xi_{p}^{-}-M^{0}\right) u_{0}^{(p)}=0, p=1, \ldots, l \\
& \left(\xi_{p}^{-}-M^{0}\right) u_{1}^{(p)}+\left(\frac{\partial}{\partial x_{k}}-M_{0}\left(D_{t}, D_{x^{*}}\right)-M_{1}\left(x, t ; \lambda_{0}, i \eta_{0}\right)\right) u_{0}^{(p)}=0 \\
& \left(\xi_{p}^{-}-M^{0}\right) u_{j+1}^{(p)}+\left(\frac{\partial}{\partial x_{k}}-M_{0}\left(D_{t}, D_{x^{\prime}}\right)-M_{1}\left(x, t ; \lambda_{0}, i \eta_{0}\right)\right) u_{j}^{(p)}=f_{j}^{(p)} \\
& \begin{array}{l}
p=1, \ldots, l \\
j=1,2, \ldots,
\end{array}
\end{aligned}
$$

and

$$
\left.\sum_{p=1}^{l} P_{0} u_{0}^{(p)}\right|_{x_{k}=0}=0
$$




$$
\begin{aligned}
& \left.\sum_{p=1}^{l}\left(P_{0} u_{1}^{(p)}+P_{1}\left(x^{\prime}, t\right) u_{0}^{(p)}\right)\right|_{x_{k}=0}=0 \\
& \left.\sum_{p=1}^{l}\left(P_{0} u_{j}^{(p)}+P_{1}\left(x^{\prime}, t\right) u_{j-1}^{(p)}\right)\right|_{x_{k}=0}=g_{j}
\end{aligned}
$$

where

$$
\begin{array}{r}
f_{j}^{(p)}=\sum_{\substack{i+s=j+1 \\
s<j}} M_{i}\left(x, t ; \lambda_{0}, i \eta_{0}\right) u_{s}^{(p)}+\sum_{\substack{i+s=j \\
s<j}} M_{i}\left(x, t, D_{\imath}, D_{x^{\prime}}\right) u_{s}^{(p)} \\
\substack{p=1, \ldots, l \\
j=1,2, \ldots,}
\end{array}
$$

and

$$
g_{j}=-\left.\sum_{\substack{i+s=j-1 \\ i<2}} \sum_{p=1}^{l} P_{i}\left(x^{\prime}, t\right) u_{s}^{(p)}\right|_{x_{k}=0}, \quad j=1,2, \ldots
$$

From (2.8) and (2.11), we have

$$
\begin{aligned}
& u_{0}^{(p)}=\sigma_{0}^{(p)}(x, t) h_{p}, p=1,2, \ldots, l, \quad \text { and } \\
& \left.\sum_{p=1}^{l} u_{0}^{(p)}\right|_{x_{k}=0}=\sum_{p=1}^{l^{\prime}} \tilde{\sigma}_{0}^{(p)}\left(x^{\prime}, t\right) z_{p},
\end{aligned}
$$

where $\sigma_{0}^{(p)}$ and $\tilde{\sigma}_{0}^{(p)}$ are scalar functions. Next, we seek $u_{1}^{(p)}$ satisfied with (2.9) and (2.12). We can find the particular solution $\dot{u}_{1}^{(p)}$ of (2.9), if $u_{0}^{(p)}$ are satisfied with

$$
\omega_{p} \cdot\left(\frac{\partial}{\partial x_{k}}-M_{0}\left(D_{t}, D_{x^{\prime}}\right)-M_{1}\left(x, t ; \lambda_{0}, i \eta_{0}\right)\right) u_{0}^{(p)}=0
$$

that is,

$$
\left(\frac{\partial}{\partial x_{k}}-a_{p}\left(D_{t}, D_{x^{\prime}}\right)-b_{p}(x, t)\right) \sigma_{0}^{(p)}=0, \quad p=1, \ldots, l,
$$

where

$$
a_{p}\left(D_{t}, D_{x^{\prime}}\right)=\omega_{p} \cdot M_{0} h_{p} \quad \text { and } \quad b_{p}=\omega_{p} \cdot M_{1} h_{p}
$$

The Cauchy Kowalweski's theorem guarantees the existence of the analytic solution $\sigma_{0}^{(p)}$ of (2.16) with the intial conditions (2.15). Here we note that $\tilde{\sigma}_{0}^{(p)}\left(x^{\prime}, t\right), \quad p=1,2, \ldots, l^{\prime}$, remain undetermined. 
From above reasoning we obtain the general solution of (2.9) as follows

$$
u_{1}^{(p)}=\sigma_{1}^{(p)}(x, t) h_{p}+\stackrel{\circ}{u}_{1}^{(p)}, \quad p=1, \ldots, l,
$$

where $\dot{u}_{1}^{(p)}$ is the particular solution of (2.9). Next, we determine $\tilde{\sigma}_{0}^{(p)}\left(x^{\prime}, t\right), \quad p=1, \ldots, l^{\prime}$, so that $\left.u_{1}^{(p)}\right|_{x_{k}=0}$ verify (1.12). We put $H=$ $\left(h_{1}, h_{2}, \ldots, h_{p}\right), \sigma_{j}={ }^{t}\left(\sigma_{j}^{(1)}, \ldots, \sigma_{j}^{(l)}\right), \quad \tilde{\sigma}_{j}={ }^{t}\left(\tilde{\sigma}_{j}^{(1)}, \ldots, \tilde{\sigma}_{j}^{\left(l^{\prime}\right)}\right)$ and $Z=\left(z_{1}, z_{2}, \ldots\right.$, $\left.z_{l^{\prime}}\right)$. Inserting (2.17) into (2.12), we obtain

$$
P_{0} H \sigma_{1}\left(x^{\prime}, 0, t\right)+\left.\sum_{p=1}^{l}\left(P_{0} \dot{\leftrightarrow}_{1}^{(p)}+P_{1} u_{0}^{(p)}\right)\right|_{x_{k}=0}=0 .
$$

From the assumption, the rank of $P_{0} H$ is $l-l^{\prime}$. Hence there are left nullvectors $r_{1}, \ldots, r_{l^{\prime}}$ of $P_{0} H$. We write $R={ }^{\prime}\left(r_{1}, \ldots, r_{l^{\prime}}\right), l^{\prime} \times m$ matrix. If $\dot{u}_{1}^{(p)}$ and $u_{0}^{(p)}$ verifies

$$
\left.R \cdot \sum_{p=1}^{l}\left(P_{0} \stackrel{\circ}{u}_{1}^{(p)}+P_{1}\left(x^{\prime}, t\right) u_{0}^{(p)}\right)\right|_{x_{k}=0}=0,
$$

there exists a solution $\sigma_{1}\left(x^{\prime}, 0, t\right)$ of (2.18). We express (2.19) as an equation of $\tilde{\sigma}_{0}$. To do so, we must solve $\stackrel{\circ}{1}_{1}^{(p)}$ explicitly from (2.9). We use operators $Q^{ \pm}$defined in (1.2). We put $M^{ \pm}=Q^{ \pm} M$. We note that $Q^{ \pm}$is an orthogonal projection into $E_{0}^{ \pm}\left(\lambda_{0}, i \eta_{0}\right)$ along $E_{0}^{\mp}\left(\lambda_{0}, i \eta_{0}\right)$. Since $Q^{-} \dot{\varkappa}_{1}^{(p)}$ is in $E_{0}^{-}, R P_{0} Q^{-} \dot{\varkappa}_{1}^{(p)}=0$. Hence by virtue of (2.9), we obtain

$$
Q^{+} \stackrel{\leftrightarrow}{u}_{1}^{(p)}=\left(\xi_{p}^{-}-M^{+}\right)^{-1} Q^{+}\left(M_{0}\left(D_{t}, D_{x^{\prime}}\right)+M_{1}\left(x, t, \lambda_{0}, i \eta_{0}\right)\right) u_{0}^{(p)}
$$

Noting that

$$
\left(\xi_{p}^{-}-M^{+}\right)^{-1} Q^{+}=\frac{1}{2 \pi i} \oint_{\Gamma_{+}}\left(\xi_{p}^{-}-\xi\right)^{-1}\left(\xi-M^{0}\right)^{-1} \mathrm{~d} \xi
$$

and

$$
\left(\xi_{p}^{-}-\xi\right)^{-1} h_{p}=\left(M^{-}-\xi\right)^{-1} h_{p} \quad \text { for } \xi \in \Gamma_{+}, p=1, \ldots, l,
$$

we have by virtue of (2.14) and (2.20),

$$
\left.\sum_{p=1}^{l} Q^{+} u_{1}^{(p)}\right|_{x_{k}=0}=\frac{1}{2 \pi i} \oint_{\Gamma_{+}}\left(\xi-M_{0}\right)^{-1}\left\{M_{0}\left(D_{t}, D_{x^{\prime}}\right)+\right.
$$




$$
\begin{gathered}
\left.+M_{1}\left(x^{\prime}, 0, t ; \lambda_{0}, i \eta_{0}\right)\right\} \\
\times\left(M^{-}-\xi\right)^{-1} \mathrm{~d} \xi \sum_{p=1}^{l} h_{p} \sigma_{0}^{(p)}\left(x^{\prime}, 0, t\right) \\
=\frac{1}{2 \pi i} \oint_{\Gamma_{+}}\left(\xi-M^{0}\right)^{-1}\left\{A_{k}^{-1}(0,0) \frac{\partial}{\partial t}+A_{k}^{-1} \sum_{j=1}^{k-1} A_{j}(0,0) \frac{\partial}{\partial x_{j}}+M_{1}\right\} \\
\cdot\left(\xi-M^{-}\right)^{-1} \mathrm{~d} \xi \times Z \cdot \tilde{\sigma}_{0}\left(x^{\prime}, t\right),
\end{gathered}
$$

which implies with (2.19),

$$
T_{0} \frac{\partial}{\partial t} \tilde{\sigma}_{0}+\sum_{j=1}^{k-1} T_{j} \frac{\partial}{\partial x_{j}} \tilde{\sigma}_{0}+S\left(x^{\prime}, t\right) \tilde{\sigma}_{0}=0
$$

where, $\quad T_{0}=R P_{0} \Lambda Z, \Lambda=\frac{1}{2 \pi i} \oint_{\Gamma_{+}}\left(\xi-M^{0}\right)^{-1} A_{k}^{-1}(0,0)\left(M^{-}-\xi\right) \mathrm{d} \xi$.

and

$$
\begin{array}{r}
T_{j}=\frac{1}{2 \pi i} \oint_{\Gamma_{+}} R P\left(\xi-M^{0}\right)^{-1} A_{k}^{-1}(0,0) A_{j}(0,0)\left(M^{-}-\xi\right)^{-1} Z \mathrm{~d} \xi, \\
j=1, \ldots, k-1,
\end{array}
$$

are $l^{\prime} \times l^{\prime}$ constant martices, and $S(x, t)$ is a $l^{\prime} \times l^{\prime}$ matrix with elements analytic in $\left(x^{\prime}, t\right)$. From the assumption (ii) of [A.III] it follows that the $l^{\prime} \times l^{\prime}$ matrix $T_{0}$ is non singular. Hence the exists non trivial solution $\tilde{\sigma}_{0}\left(x^{\prime}, t\right)$ of $(2.21)$ and therefore we have a solution $\sigma_{1}\left(x^{\prime}, 0, t\right)$ of (2.18) such that

$$
H \sigma_{1}\left(x^{\prime}, 0, t\right)=Z \tilde{\sigma}_{1}\left(x^{\prime}, t\right)+H \dot{\sigma}_{1}\left(x^{\prime}, t\right)
$$

where $\dot{\sigma}_{1}\left(x^{\prime}, t\right)$ is a particular solution of (2.18).

Summarising the above result, we find $u_{0}^{(p)}$ as follows,

$$
\begin{aligned}
& u_{0}^{(p)}(x, t)=\sigma_{0}^{(p)}(x, t) h_{p}, \quad p=1,2, \ldots, l \\
& H \sigma_{0}\left(x^{\prime}, 0, t\right)=Z \tilde{\sigma}_{0}\left(x^{\prime}, t\right),
\end{aligned}
$$

here $\sigma_{0}(x, t)={ }^{t}\left(\sigma_{0}^{(1)}(x, t), \ldots, \sigma_{0}^{(l)}(x, t)\right)$ is a solution of (2.16) and its initial value $\tilde{\sigma}_{0}\left(x^{\prime}, t\right)$ is a solution of (2.21). Next, we have $u_{1}^{(p)}(x, t)$ as follows, 


$$
\begin{gathered}
u_{1}^{(p)}(x, t)=\sigma_{1}^{(p)}(x, t) h_{p}+\stackrel{\leftrightarrow}{u}_{1}^{(p)}(x, t), \quad p=1,2, \ldots, l, \\
H \sigma_{1}(x, 0, t)=Z \tilde{\sigma}_{1}\left(x^{\prime}, t\right)+H \stackrel{\circ}{\sigma}_{1}\left(x^{\prime}, t\right),
\end{gathered}
$$

where $\stackrel{\leftrightarrow}{u}_{1}^{(p)}$ is a particular solution of $(2.9)$ and $\dot{\sigma}_{1}\left(x^{\prime}, t\right)$ is a particular solution of (2.18), and $\sigma_{1}(x, t)={ }^{t}\left(\sigma_{1}^{(1)}, \ldots, \sigma_{1}^{(l)}\right)$ and $\tilde{\sigma}_{1}\left(x^{\prime}, t\right)={ }^{t}\left(\sigma_{1}^{(1)}, \ldots\right.$, $\left.\sigma_{1}^{\left(l^{\prime}\right)}\right)$ are arbitrary functions in order to construct $u_{2}^{(p)}(x, t), p=1, \ldots, l$.

Generally, we put $u_{s}^{(p)}(x, t)$ such that

$$
u_{s}^{(p)}(x, t)=\sigma_{s}^{(p)}(x, t) h_{p}+\dot{u}_{s}^{(p)}(x, t), \quad p=1, \ldots, l,
$$

and

$$
H \sigma_{s}\left(x^{\prime}, 0, t\right)=Z \tilde{\sigma}_{s}(x, t)+H \dot{\sigma}_{s}\left(x^{\prime}, t\right) .
$$

where $\stackrel{\circ}{u}_{s}^{(p)}$ is a particular solution of (2.10) with $j=s-1$ and $H \dot{\sigma}_{s}\left(x^{\prime}, t\right)$ is a particular solution of (2.13) with $j=s$. Then if we choose $\sigma_{s}^{(p)}(x, t)$ and $\tilde{\sigma}_{s}\left(x^{\prime}, t\right)$ such that,

$$
\left(\frac{\partial}{\partial x_{k}}-a_{p}\left(D_{t}, D_{x^{\prime}}\right)-b_{p}(x, t)\right) \sigma_{s}^{(p)}=f_{s}^{(p)}(x, t), \quad p=1, \ldots, l,
$$

and

$$
\left(T_{0} \frac{\partial}{\partial t}+\sum_{j=1}^{k-1} T_{j} \frac{\partial}{\partial x_{j}}+S\left(x^{\prime}, t\right)\right) \tilde{\sigma}_{s}=\tilde{g}_{s}\left(x^{\prime}, t\right)
$$

where $\tilde{f}_{s}^{(p)}=\omega_{p} \cdot f_{s}^{(p)}-\omega_{p} \cdot\left(\frac{\partial}{\partial x_{k}}-M_{0}\left(D_{t}, D_{x}\right)-M_{1}\left(x, t ; \lambda_{0}, i \eta\right)\right) u_{s}^{(p)}(x, t)$ and $\tilde{g}_{s}=R g_{s+1}-\sum_{p=1}^{l} R P_{1}\left(x^{\prime}, t\right) \dot{u}_{s}^{(p)}\left(x^{\prime}, 0, t\right)-R P_{1}(x, t) H \dot{\sigma}_{s}\left(x^{\prime}, t\right)$, we can construct successively $u_{s+1}^{(p)}(x, t)$ satisfying (2.10) with $j=s$ and (2.13) with $j=s+1$.

We put

$$
u(x, t)=\sum_{j=0}^{N} n^{-j} \sum_{p=1}^{l} \exp \left\{n\left(\xi_{p}^{-} \cdot x_{k}+\lambda_{0} t-i x^{\prime} \eta_{0}\right)\right\} u_{j}^{(p)}(x, t) .
$$

Then if $N$ is sufficiently large, it is obvious that $u(x, t)$ violates (2.3). Thus the proof of our theorem is complete. 
Remark. In above statement, we assumed that the matrix $M_{0}$ has simple eigen values. But this is not essential. In fact, it follows from the assumption (i) of [A. III] that, if $\xi_{p}^{-}$is an eigen value of multiplicity $v_{p}$, there exist the number $v_{p}$ of linearly independent eigen vectors corresponding to $\xi_{p}^{-}$. We can repeat the above reasoning by use of those eigen evctors.

\section{Department of Applied Mathematics AND Physics, Kyoto Univrsity}

\section{References}

[1] R. Beals; Mixed boundary value problem for non strict hyperbolic equations, Bull. A. M. S. (1972), 520-521.

[2] J. Chazarain; Problèmes de Cauchy abstraits et application á quelques problèmes mixtes, J. Funct. Analysis Vol. 7, (1971), 386-446.

[3] M. Ikawa; Mixed problem for the wave equation with an oblique derivative boundary condition, Osaka J. Math. Vol. 7. (1970), 495-525.

[4] V.J. Ivrii; The Cauchy problem for non strict hyperbolic equations, Dokl. Akad. Nauk, Vol. 197. (1971), 483-486.

[5] R. Hersh; Mixed problem in several variables, J. Math. Mech. Vol. 12. (1963), 317-334.

[6] R. Hersh; Boundary condition for equations of evolution, Arch. Rat. Mech. Anal. Vol. 16. (1964), 243-264.

[7] R. Hersh; On surface waves with finite and infinite speed of propagation, Arch. Rat. Mech. Anal. Vol. 19. (1965), 308-316.

[8] K. Kajitani; Sur la condition nécessaire du problème mixte bien posé pour les systèmes hyperboliques, to appear in Rubl. RIMS, (1973).

[9] K. Kajitani; Strongly hyperbolic systems with variable coefficients, to appear in Rubl. RIMS, Vol. 9. (1974), 597-612.

[10] K. Kasahara; On weak well posedness of mixed problem for hyperbolic systems, Rubl. RIMS, Vol. 6. (1971), 503-514.

[11] P. Lax; Asymptotic solution of oscillatory intial value problems, Duke Math. J. Vol. 24. (1957), 627-646.

[12] S. Mizohata; Some remarks on the Cauchy problem, J. Math. Kyoto Univ. Vol. 1. (1961), 109-127.

[13] R. Sakamoto; Hyperbolic mixed problems with constant coefficients, to appear.

[14] T. Shirota; On propagation speed of hyperbolic mixed boundary conditions, J. Fac. Sci. Hokkaido Univ. Vol. 22. (1972), 25-31. 